\title{
Laboratory evidence for volume-dominated nucleation of ice in supercooled water microdroplets
}

\author{
D. Duft and T. Leisner \\ Institut für Physik, Technische Universität Ilmenau, 98684 Ilmenau, Germany \\ Received: 31 March 2004 - Published in Atmos. Chem. Phys. Discuss.: 7 June 2004 \\ Revised: 30 August 2004 - Accepted: 3 September 2004 - Published: 4 October 2004
}

\begin{abstract}
We report on measurements of the rate of homogeneous ice nucleation in supercooled water microdroplets levitated in an electrodynamic balance. By comparison of the freezing probability for droplets of radius $49 \mu \mathrm{m}$ and $19 \mu \mathrm{m}$, we are able to conclude that homogeneous freezing is a volume-proportional process and that surface nucleation might only be important, if at all, for much smaller droplets.
\end{abstract}

\section{Introduction}

It is well known that supercooled liquid cloud and fog droplets are often encountered in the atmosphere at temperatures far below their equilibrium melting temperature and sometimes even below the frost point (Sassen and Dodd, 1988; Heymsfield and Sabin, 1989; Heymsfield and Miloshevich, 1993). While most of the atmospheric freezing processes occur heterogeneously via ice nuclei, homogeneous ice nucleation occurs below about $-38^{\circ} \mathrm{C}$ where it forms the ultimate limit for supercooling. It has been identified to be important in the formation of high altitude cirrus- and polar stratospheric clouds and in the glaciation of tropical thunderstorm clouds. While in the former case sub- micron droplets freeze homogeneously, in the latter case droplets in the $10 \mu \mathrm{m}$ to $30 \mu \mathrm{m}$ diameter range may be involved.

Rates for the homogeneous freezing of water ice have been measured previously in laboratory experiments by Taborek (1985) using emulsion samples, by DeMott and Rogers (1990) in cloud chambers, by Wood et al. (2002) in droplet trains and in levitated single particles by Krämer et al. (1996, 1999) and Stöckel et al. (2002). The droplets in these experiments were between $3 \mu \mathrm{m}$ and $300 \mu \mathrm{m}$ in diameter and in all cases the data were interpreted as volume-nucleation rates,

Correspondence to: Thomas Leisner

(thomas.leisner@tu-ilmenau.de) i.e. the freezing rate was assumed to be proportional to the droplet volume.

These data have been reanalysed recently by Tabazadeh et al. (2002a, b) and Djikaev et al. (2002, 2003). They find evidence that freezing nucleation in a small layer at the droplet surface might be dominant under atmospheric conditions. This would have important consequences, as the surface of cloud and aerosol particles is much more prone to anthropogenic and natural modification than the volume material. They have been objected by (Kay et al., 2003) from theoretical considerations and from the analysis of the laboratory data. It is the purpose of our investigation to provide new and improved laboratory data to quantify the importance of surface nucleation in atmospheric processes.

For a quantitative description of homogeneous nucleation, classical nucleation theory is usually invoked (Volmer and Weber, 1925; Zeldovich, 1942; Kelton, 1991). It is assumed that the formation of a single ice crystallite of a certain size (a germ or nucleus) initiates the crystallization of the whole droplet. The freezing process is then governed by a first order rate equation:

$$
\begin{aligned}
& d P_{u}(t) / d t=-P_{u} \cdot J(T) \cdot V \quad \text { or } \\
& -d \ln P_{u}(t) / d t=J(T) \cdot V
\end{aligned}
$$

Here $P_{u}(t)$ is the probability for the droplet to be still unfrozen at time $\mathrm{t}$ after being quenched at time $t=0$ from high temperature to the temperature $T$. The freezing rate is proportional to the droplet volume and the proportionality constant $J(T)$ is called the (volume-) nucleation rate. Experimentally, $\ln \left(P_{u}(t)\right)$ is usually measured as a function of time. If $V$ and $T$ are constant, this should yield a linear decline, the negative slope of which is the freezing rate and is identified with $J(T) \cdot V$.

In order to include surface freezing, Eq. (1) should be replaced by the more precise form

$-d \ln P_{u}(t) / d t=J_{V}(T) \cdot V_{V}+J_{S}(T) \cdot V_{S}$ 


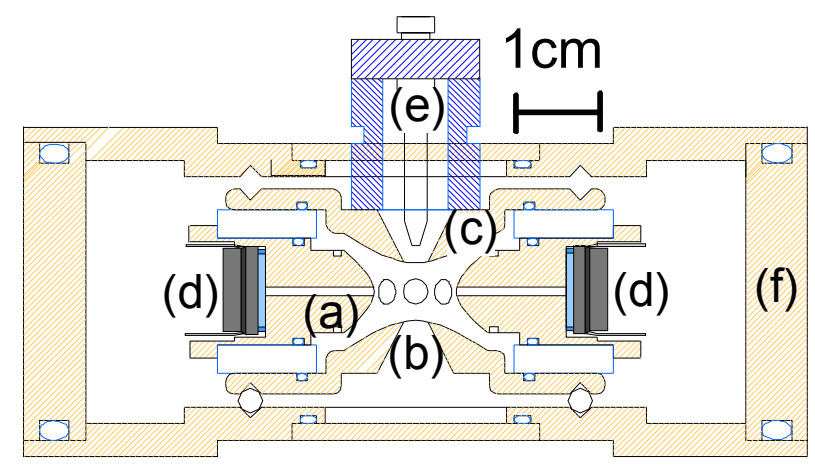

Fig. 1. Schematic vertical cross section through the levitator, (a) cooled central electrode, (b) bottom and (c) top endcap electrode, (d) linear CCD detectors, (e) droplet generator and injector, (f) vacuum chamber.

where $J_{V}$ is the volume nucleation rate, $V_{V}$ is the bulk volume, $J_{S}$ is the surface nucleation rate and $V_{S}$ is the surface volume, i.e. $V_{S}=4 \cdot \pi \cdot r^{2} \cdot d_{S}$ with $r$ being the droplet radius and $d_{S}$ the thickness of the surface layer that is governed by surface freezing. If, for simplicity, we assume spherical symmetry and $V=V_{V} \gg V_{S}$, we can bring this into the form:

$-d \ln P_{u} / d t=J_{V} \cdot V \cdot\left(1+\frac{1}{r} \cdot \frac{3 d_{S} \cdot J_{S}}{J_{V}}\right)=J_{V} \cdot V \cdot\left(1+\frac{r_{c}}{r}\right)$

The correction due to surface nucleation is given by the second addend in the brackets and is inversely proportional to the droplet radius. The proportionality constant $r_{c}=3 d_{S} J_{S} / J_{V}$ has the dimension of a length and can be interpreted as the critical radius of a droplet for which surfaceand volume- nucleation are equally important. For larger droplets, volume nucleation prevails and vice versa. In order to assess the role of surface nucleation in atmospheric processes, the typical droplet size of interest has to be compared to $r_{c}$.

Fortunately, $r_{c}$ can be determined directly by experiment. It is sufficient to measure $\alpha$, the ratio of the freezing rates of two different monodisperse droplet ensembles of radii $r_{1}$ and $r_{2}$ respectively compared to the volume ratio of the two ensembles:

$\alpha=\frac{V_{1}}{V_{2}} \cdot \frac{\left(d \ln P_{u} / d t\right)_{2}}{\left(d \ln P_{u} / d t\right)_{1}}=\frac{\left(1+r_{c} / r_{2}\right)}{\left(1+r_{c} / r_{1}\right)} \quad$ or

$r_{c}=\frac{\alpha-1}{1 / r_{2}-\alpha / r_{1}}$

Here we report on such a measurement performed for droplets of radius $r_{1}=49 \mu \mathrm{m}$ and $r_{2}=19 \mu \mathrm{m}$ at a temperature of $237.1 \mathrm{~K}$ corresponding to a supercooling of $36.1 \mathrm{~K}$.

\section{Experimental}

The freezing of many individual droplets of each size was observed inside an electrodynamic levitator which was kept at

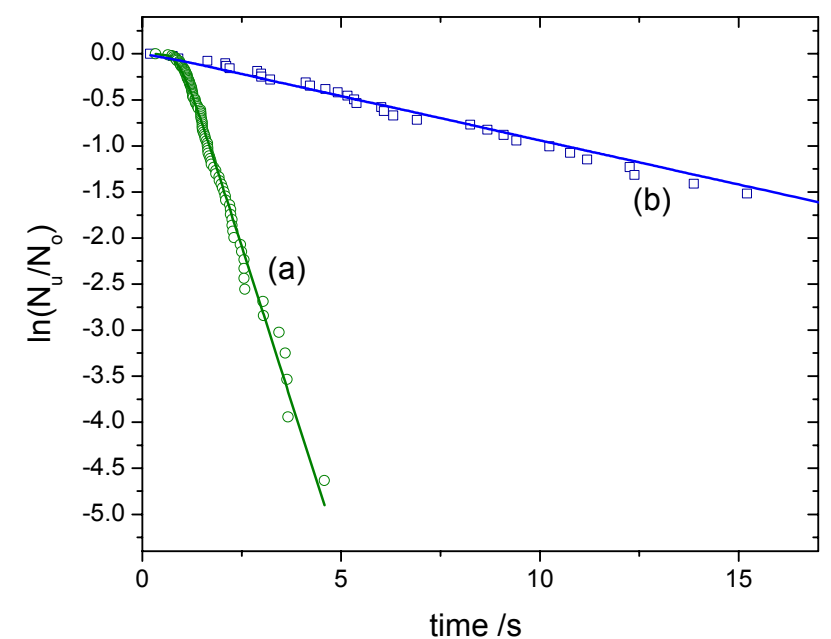

Fig. 2. Logarithm of the fraction of unfrozen droplets as a function of time after injection in the droplet levitator. (a) open circles: large droplets with an average radius $r_{1}=49 \mu \mathrm{m}$, (b) open squares: small droplets with an average radius $r_{2}=19 \mu \mathrm{m}$.

the low temperature of interest throughout the experiment. Temperature variations were below $20 \mathrm{mK}$ (single sigma). The method of electrodynamical levitation was reviewed by Davis (1997), details of our experimental setup have been given elsewhere (Duft et al., 2002; Duft and Leisner, 2004). Briefly, the levitator is of hyperboloidal type as proposed for microparticles by Fischer (1959) and Wuerker and Langmuir (1959) consisting of a rotationally symmetric torus electrode and two endcap electrodes (cf. Fig. 1a, b, c). The central torus electrode is formed to serve as a climate chamber and carries various ports for optical access to the droplets. It also embodies two linear CCD (charge coupled device) array detectors (Fig. 1d) to measure the angular resolved intensity of light scattered by the droplet from a HeNe laser beam (phase function measurement). As Duft and Leisner (2004) have shown recently, the analysis of the phase function allows to determine the size and refractive index of the droplets in real time. The trap is housed inside a vacuum chamber (Fig. 1f) for thermal insulation and connected to a liquid nitrogen cooled micro- cryostat for temperature adjustment. Great care has been taken to guarantee temperature uniformity over the trap volume.

The two size classes of droplets of different diameter were generated and injected into the levitator by a piezoelectric droplet on demand generator (Fig. 1e) which was operated at room temperature at two different driving pulse amplitudes. The droplets of the first class (low driving pulse amplitude) had a diameter $d_{1}=98 \pm 1 \mu \mathrm{m}$ while the droplets of class two are straggler droplets which occur at high driving pulse amplitude and were characterized by a size of $d_{2}=38 \pm 0.5 \mu \mathrm{m}$. At the low temperatures of our experiment, evaporation of the droplets during the short time interval until freezing occured was negligible. In order to be levitated 
Table 1. Comparison of the freezing probabilities of water droplets of different size.

\begin{tabular}{llll}
\hline Temperature $\mathrm{T}=237.1 \mathrm{~K}$ & Size class 1 & Size class 2 & Ratio (class1/class2) \\
\hline Radius $[\mu \mathrm{m}]$ & $49 \pm 0.5$ & $19 \pm 0.25$ & $2.58 \pm 0.05$ \\
Volume $\left[\mu \mathrm{m}^{3}\right]$ & $(4.93 \pm 0.15) \times 10^{5}$ & $(2.87 \pm 0.1) \times 10^{4}$ & $17.2 \pm 0.8$ \\
Freezing rate $\left[\mathrm{s}^{-1}\right]$ & $1.35 \pm 0.05$ & $(8.2 \pm 0.3) \times 10^{-2}$ & $16.5 \pm 0.6$ \\
Volume nucleation rate $\left[\mathrm{cm}^{-3} \mathrm{~s}^{-1}\right]$ & $(2.75 \pm 0.15) \times 10^{6}$ & $(2.85 \pm 0.15) \times 10^{6}$ & $0.96 \pm 0.07$ \\
Surface nucleation rate $\left[\mathrm{cm}^{-2} \mathrm{~s}^{-1}\right]$ & $(4.47 \pm 0.19) \times 10^{3}$ & $(1.81 \pm 0.075) \times 10^{3}$ & $2.47 \pm 0.15$ \\
Number of droplets examined & 103 & 41 & \\
\hline
\end{tabular}

electrodynamically, each droplet carries a specific charge of about $10^{-4} \mathrm{C} / \mathrm{kg}$. In the case of the larger droplets, this corresponds to roughly $10^{6}$ additional $\mathrm{H}_{3} \mathrm{O}^{+}$ions distributed homogeneously around the surface, which is made up by about $1.5 \times 10^{11}$ molecules. These numbers indicate that there are plenty of undisturbed sites available for surface nucleation. In previous experiments by Krämer et al. (1999), the surface charge was varied by a factor of three and no detectable influence on the rate of homogeneous freezing was found for both positively and negatively charged droplets.

Freezing phase transitions in droplets can easily be detected by light scattering measurements. The phase function of a liquid droplet shows well resolved intensity minima and maxima as expected from Mie theory for a homogeneous sphere, while the frozen ice particles generate a highly irregular light scattering pattern due to multiple scattering on the grain boundary network. An automated phase function analysis is used to determine the time of freezing for each droplet with a time resolution of $10 \mathrm{~ms}$. The freezing rate of the droplets is determined by plotting the logarithm of the frequency of occurrence of unfrozen droplets versus the time after injection. The results for both size classes are given for a temperature of $T=237.1 \mathrm{~K}$ in Fig. 2.

The data do not lie exactly on a straight line as expected both from Eqs. (2) and (3) but rather start off with a horizontal slope. This is especially evident in Fig. 2a and reflects the fact that the droplets are injected into the levitator at room temperature and then have to accommodate to the trap temperature. After this thermalization, which takes longer time for larger droplets, the curves assume a constant slope. From this slope, the freezing rate is deduced for both sizes. For increased accuracy, this is done by fitting a solution to a differential equation, which takes the initial cooling period into account. Details of this analysis will be given in a subsequent publication. The fitted curve in Fig. $2 \mathrm{~b}$ may seem to be too flat. This is due to the fact that in Fig. $2 b$ data points at longer times $(t>15 \mathrm{~s})$ are omitted for clarity. These data points have been taken into account in the fitting procedure. The result of the measurements is summarized in Table 1.

\section{Discussion}

If we interpret our data in the framework of volume freezing, we arrive for the larger size class at a nucleation rate
$J_{V}=(2.8 \pm 0.15) \times 10^{6} \mathrm{~cm}^{-3} \mathrm{~s}^{-1}$, a value which is in very good agreement with our previous measurements (Krämer et al., 1999) and the STO emulsion data of Taborek (1995). Comparing the two size classes, we find that the volume ratio $V_{1} / V_{2}$ and the freezing rate ratio lie within the limits of error of each other. Accordingly, the volume nucleation rates in the two classes are equivalent. If, for comparison, we divide the respective freezing rates by the surface area of the droplets, the resulting surface nucleation rates are about a factor of 2.5 apart for the two size classes. We conclude that within the investigated size range, the freezing rate of supercooled water scales with the droplet volume and freezing therefore is a volume-dominated process. Surface freezing is not needed to explain the results. If we want to stress our data somewhat, we can determine from Eq. (4) the droplet radius below which surface nucleation becomes important, but this critical radius carries a large error bar. Any value between $r_{c}=0$ and $r_{c}=4 \mu \mathrm{m}$ is consistent with our data.

Our results suggest that, for droplet radii of some ten micrometers, as they were predominantly used in previous experiments, homogeneous nucleation proceeds by a volume proportional rate. It cannot be ruled out, however, that surface nucleation is important for much smaller droplets below $1 \mu \mathrm{m}$, as they are typical for aerosol droplets which are more likely to freeze homogeneously in the atmosphere. By extending our experiments to smaller droplets we might be able to lower the upper limit for $r_{c}$ into this size regime.

The main argument that supported surface nucleation was based on a reanalysis of the laboratory data which yielded varying volume-nucleation rates. These experimental data seemed more consistent if interpreted as surface nucleation rates. In the light of our results we rather assume that the differences in nucleation rate measurements were related to experimental problems with the assessment of droplet volume and temperature instead. This has been confirmed for the data presented by Stöckel et al. (2002). Revised and extended data by Stöckel et al. (2004) ${ }^{1}$ support this point of view. 
Acknowledgements. The work was partly funded by the German Science Foundation DFG under contract no. LE834/1 We are indebted to Peter Stöckel, Hermann Vortisch and Helmut Baumgärtel for ongoing discussions.

Edited by: S. Martin

\section{References}

Davis, E. J.: A History of Single Particle Levitation, Aero. Sci. Technol., 26, 212-254, 1997.

DeMott, P. J. and Rogers, D. C.: Freezing nucleation rates of dilute solution droplets, J. Atmos. Sci., 47, 1056-1064, 1990.

Djikaev, Y. S., Tabazadeh, A., Hamill, P., and Reiss, H.: Thermodynamic Conditions for the Surface-Stimulated Crystallization of Atmospheric Droplets, J. Phys. Chem. A, 106, 10247-10253, 2002.

Dijkaev, Y. S., Tabazadeh, A., and Reiss, H.: Thermodynamics of crystal nucleation in multicomponent droplets: Adsorption, dissociation, and surface-stimulated nucleation, J. Chem. Phys., 118, 6572-6581, 2003.

Duft, D., Lebius, H., Huber, B. A., Guet, C., and Leisner, T.: Shape Oscillations and Stability of Charged Microdroplets, Phys. Rev. Lett., 89, art. no. 084503, 1-4, 2002.

Duft, D. and Leisner, T.: The index of refraction of supercooled solutions determined by the analysis of optical rainbow scattering from levitated droplets, Int. J. Mass Spec., 233, 61-65, 2004.

Fischer, E.: The three dimensional stabilizing of charged particles in a quadropole field, Zeitschrift f. Physik, 156, 1-26, 1959.

Heymsfield, A. J. and Miloshevich, L. M.: Relative humidity and temperature influences on cirrus formation and evolution: $\mathrm{Ob}-$ servations from wave clouds and FIRE-II, J. Atmos. Sci., 50, 2335-2353, 1993.

Heymsfield, A. J. and Sabin, R. M.: Cirrus crystal nucleation by homogeneous freezing of solution droplets, J. Atmos. Sci., 46, 2252-2264, 1989.

Kay, J. E., Tsemekhman, V., Larsen, B., Baker, M., and B. Swanson: Comment on evidence for surface-initiated homogeneous nucleation, Atmos. Chem. Phys., 3, 1439-1443, 2003,

SRef-ID: 1680-7324/acp/2003-3-1439
Kelton, K. F.: Crystal Nucleation in Liquids and Glasses, Solid State Phys., 25, 75-177, 1991.

Krämer, B., Schwell, M., Hübner, O., Vortisch, H., Leisner, T., Rühl, E., Baumgärtel, H., and Wöste, L.: Homogeneous Ice Nucleation Observed in Single Levitated Micro Droplets, Ber. Bunsenges. Phys. Chem., 100, 1911-1914, 1996.

Krämer, B., Hübner, O., Vortisch, H., Wöste, L., Leisner, T., Schwell, M., Rühl, E., and Baumgärtel, H.: Homogeneous Nucleation Rates of Supercooled Water Measured in Single Levitated Microdroplets, J. Chem. Phys., 111, 6521-6527, 1999.

Pruppacher, H. and Klett, J. D.: Microphysics of Clouds and Precipitation, Kluwer Academic Publishers, 2nd Ed., Dordrecht, 1998.

Sassen, K. and Dodd, G. C. J.: Homogeneous nucleation rate for highly supercooled cirrus cloud droplets, Atmos. Sci., 45, 13571369, 1988.

Stöckel, P., Vortisch, H., Leisner, T., and Baumgärtel, H.: Homogeneous nucleation of supercooled liquid water in levitated microdroplets, J. Mol. Liq., 96-97, 153-175, 2002.

Tabazadeh, A., Djikaev, Y. S., Hamill, P., and Reiss, H.: Laboratory Evidence for Surface Nucleation of Solid Polar Stratospheric Cloud Particles, J. Phys. Chem. A, 106, 10238-10 246, 2002.

Tabazadeh, A., Djikaev, Y. S., and Reiss, H.: Surface crystallization of supercooled water in clouds, Proc. Natl. Acad. Sci. USA, 99, 15 873-15 878, 2002.

Taborek, P.: Nucleation in emulsified supercooled water, Phys. Rev. B, 32, 5902-5906, 1985.

Volmer, M. and Weber, A.: Nuclei formation in supersaturated states, Z. Phys. Chem., 119, 277-301, 1926.

Wood, S. E., Baker, M. B., and Swanson, B. D.: Instrument for studies of homogeneous and heterogeneous ice nucleation in freefalling supercooled water droplets, Rev. Sci. Instr., 73, 39883996, 2002.

Wuerker, R. F. and Langmuir, R. V.: Electrodynamic containment of charged particles, Appl. Phys., 30, 342-349, 1959.

Zeldovich, J.: Theory of the formation of a new phase. Cavitation, Zh. Eksp. Theor. Fiz., 12, 525-538, 1942. 